\title{
Automatic detection and tracking of filaments for a solar feature database
}

\author{
J. Aboudarham ${ }^{1}$, I. Scholl ${ }^{1,2}$, N. Fuller ${ }^{1}$, M. Fouesneau ${ }^{3}$, M. Galametz ${ }^{3}$, F. Gonon ${ }^{3}$, A. Maire ${ }^{3}$, and Y. Leroy ${ }^{3}$ \\ ${ }^{1}$ Laboratoire d'Etudes Spatiales et d'Instrumentation en Astrophysique (LESIA), Observatoire de Paris-Meudon, France \\ ${ }^{2}$ International Space University (ISU), Strasbourg, France \\ ${ }^{3}$ Ecole Nationale Supérieure de Physique de Strasbourg (ENSPS), Strasbourg, France
}

Received: 19 January 2007 - Revised: 31 May 2007 - Accepted: 5 June 2007 - Published: 26 February 2008

\begin{abstract}
A new method for the automatic detection and tracking of solar filaments is presented. The method addresses the problems facing existing catalogs, such as the one developed recently in the frame of the European Grid of Solar Observations (EGSO) project. In particular, it takes into account the structural and temporal evolution of filaments, differences in intensity as seen from one observation to the next, and the possibility of sudden disappearance followed by reappearance. In this study, the problem of tracking is solved by plotting all detected filaments during each solar rotation on a Carrington map and then by applying region growing techniques on those plots. Using this approach, the "fixed" positions of the envelopes in the Carrington system can be deduced. This is followed by a backward tracking of each filament by considering one full solar rotation. The resulting shifted Carrington map then enables one to follow any filament from one rotation to the next. Such maps should prove valuable for studies of the role of filaments in solar activity, notably coronal mass ejections (CMEs).
\end{abstract}

Keywords. Solar physics, astrophysics, and astronomy (Photosphere and chromosphere; Instruments and techniques)

\section{Introduction}

The first series of "Cartes Synoptiques de la Chromosphere Solaire" was published in 1928 by L. d'Azambuja. The usefulness of these maps comes from their ability to present at a glance a comprehensive view of the solar activity during a full rotation. Data used to produce these maps are obtained from Meudon full sun spectroheliograms, with data gaps filled by using Coimbra observations, since both instruments are very similar. Filaments are extracted from $\mathrm{H} \alpha$ ob-

Correspondence to: J. A boudarham

(jean.aboudarham@obspm.fr) servations, prominences obtained with Ca II K3 observations overexposed with an absorbing mask on the solar disk, faculae from Ca II K, and sunspots from Ca II K1. These solar features are then copied from solar images to synoptic maps. The synthesis is then realized on a full Carrington rotation leading to the Meudon synoptic maps.

Prior to 1998, these maps were completed manually ${ }^{1}$. After 1998, the outline of solar structures was obtained using a graphics tablet, and, once the synthesis was made, the plot of synoptic maps was automatically realized. In 2003, when the person in charge of synoptic maps production retired, this activity stopped. The only realistic way to resume this activity and therefore to continue providing the community with this powerful tool, was to fully automate this process immediately after the data acquisition.

In this paper, we concentrate on the preparation of filament maps and their tracking over time. Section 2 introduces the characteristics of the filaments as they appear in the observations used for this study. Section 3 presents the first step of this processing, namely the automatic detection of filaments. Section 4 is devoted to the description of these synoptic maps. This is followed by the description of the tracking principles in Sect. 5. Finally, Sect. 6 presents examples of scientific investigations that can benefit from the existence of such maps.

\section{Characteristics of filaments as seen in $\mathrm{H} \alpha$}

Filaments appear as the darkest features in absorption in $\mathrm{H} \alpha$ observations. They are commonly described as elongated features but in fact the limitation in contrast in Earth-based observations prohibits the use of such a definition. However, they are also frequently seen segmented in several small

\footnotetext{
${ }^{1}$ Recent synoptic maps are available in the BASS 2000 Meudon archive (http://bass2000.obspm.fr/home.php), from Carrington rotation 1824 to rotation 2008 (i.e. from 1989/12/19 to 2003/09/26).
}

Published by Copernicus Publications on behalf of the European Geosciences Union. 
parts, each of them appearing as a dark blob in $\mathrm{H} \alpha$ on the solar disk. The only unambiguous way to define a filament in terms of automatic feature recognition is to search for the darkest features in $\mathrm{H} \alpha$ observations. The shape itself may vary, not only intrinsically, but also due to variations in the transparency of the Earth's atmosphere, or due to variations of the angle between the local normal of the filament and the line of sight. During the automatic detection process, the outcome is such that they may appear with a very different shape from one image to the following one and then be identified as several filaments. They can even drift on the solar surface, especially when located close to the limb. Therefore, time tracking of filaments is extremely complicated. Any attempt to follow filaments in a sequence of images will have to deal with those inextricable distortions. While a quiescent filament may last for a few months, an active region filament may erupt shortly after it appears and then may disappear. But filament disappearance does not only occur in a violent way. "Disparition brusque" (DB, sudden disappearance) of a quiescent filament is not always final. And it has been shown that in a temporary DB, the filament remains visible at high temperatures, indicating that temporary DBs are thermal DBs (see Mouradian and Soru-Escaut, 1989, for a discussion on that topic). Checking different observations at very different temperatures in order to verify the possible presence of a filament is a tedious task. To add to the complexity of the problem, a filament can not only lose its shape, but also can disappear for a while and then re-appear later. This means that an alternative method has to be found in order to avoid this complexity when tracking filaments over time.

\section{Solar feature detection}

EGSO, the European Grid of Solar Observations, (Bentley et al., 2004) ${ }^{2}$ is a program funded by the European Community within the 5th Framework Program. Its goal was to build a virtual solar observatory with new and powerful added values for data analysis. One of its work packages was devoted to solar feature recognition. The sizeable effort made in this field was productive and successful, and codes were developed to automatically detect filaments (Fuller et al., 2005), sunspots (Zharkov et al., 2004), faculae (Benkhalil et al., 2006) and prominences.

For the purpose of this paper, only the filament detection is presented. This processing consists of two steps: image cleaning (detailed in Zharkova et al., 2003, see Sect. 3.1) followed by the filament detection itself (detailed in Fuller et al., 2005, see Sect. 3.2).

The whole process is based on full disk images of the Sun. Such data are available from different observatories around

\footnotetext{
${ }^{2}$ http://www.egso.org/
}

the world such as the Halpha observations from the Global High-Resolution Halpha Network ${ }^{3}$.

\subsection{Image cleaning}

Since an original image may contain several types of defects, a set of preliminary processes must be applied to clean it in order to avoid false features or to miss faint features.

The first processing consists of limb fitting to apply a geometrical correction to the solar shape. This first step corrects the distortion of the image due to non-exact square size pixels. It then helps to center the limb on the image. Afterwards, an intensity-based normalization is applied and consists of two more steps: correction of center-to-limb darkening as well as sky transparency variations. While spectroheliograms are obtained by scanning the Sun, some faint clouds may darken the obtained image in some places. Finally, since grains of dust may occasionally settle on the slit, those effects appear on images as very thin straight and faint lines, which have to be removed during an extra cleaning process. Intensity variations of the solar disk must be handled very carefully to avoid errors during the next step, namely the filament detection.

\subsection{Filament detection}

After this cleaning process, the real detection begins. First, the image is enhanced using a Laplacian filter. Then, seeds of filaments are detected using a local image thresholding, followed by a region growing process. Finally, a morphological closing helps to recover fainter parts of filaments. As a result, a complete geometrical description of the filament is obtained. An exhaustive list of the detected characteristics can be found in Aboudarham and Zharkov (2004). In Fig. 1, an example of filament detection obtained from a Meudon $\mathrm{H} \alpha$ spectroheliogram is presented.

As shown by Fuller et al. (2005), the comparison of the automatic detection with the manual one yields in accuracy of $89.1 \%$ for the number of filaments, and for $92.3 \%$ on their total length, indicating that mostly very small filaments are missed. They also mention that most of the failures in detection are due to filaments appearing and disappearing at the limb. This point is less critical for scientific studies, since geometrical artefacts at the edge of the Sun are so significant that one must be very cautious before using those filaments.

\section{Manual generation of Meudon Synoptic maps}

Meudon Synoptic Maps of Solar Activity provide a powerful synthesis of the visible solar activity during one Carrington rotation. Moreover, a set of such maps gives a quick overview of the evolution of the activity over time. When

\footnotetext{
${ }^{3}$ http://www.bbso.njit.edu/Research/Halpha/
} 


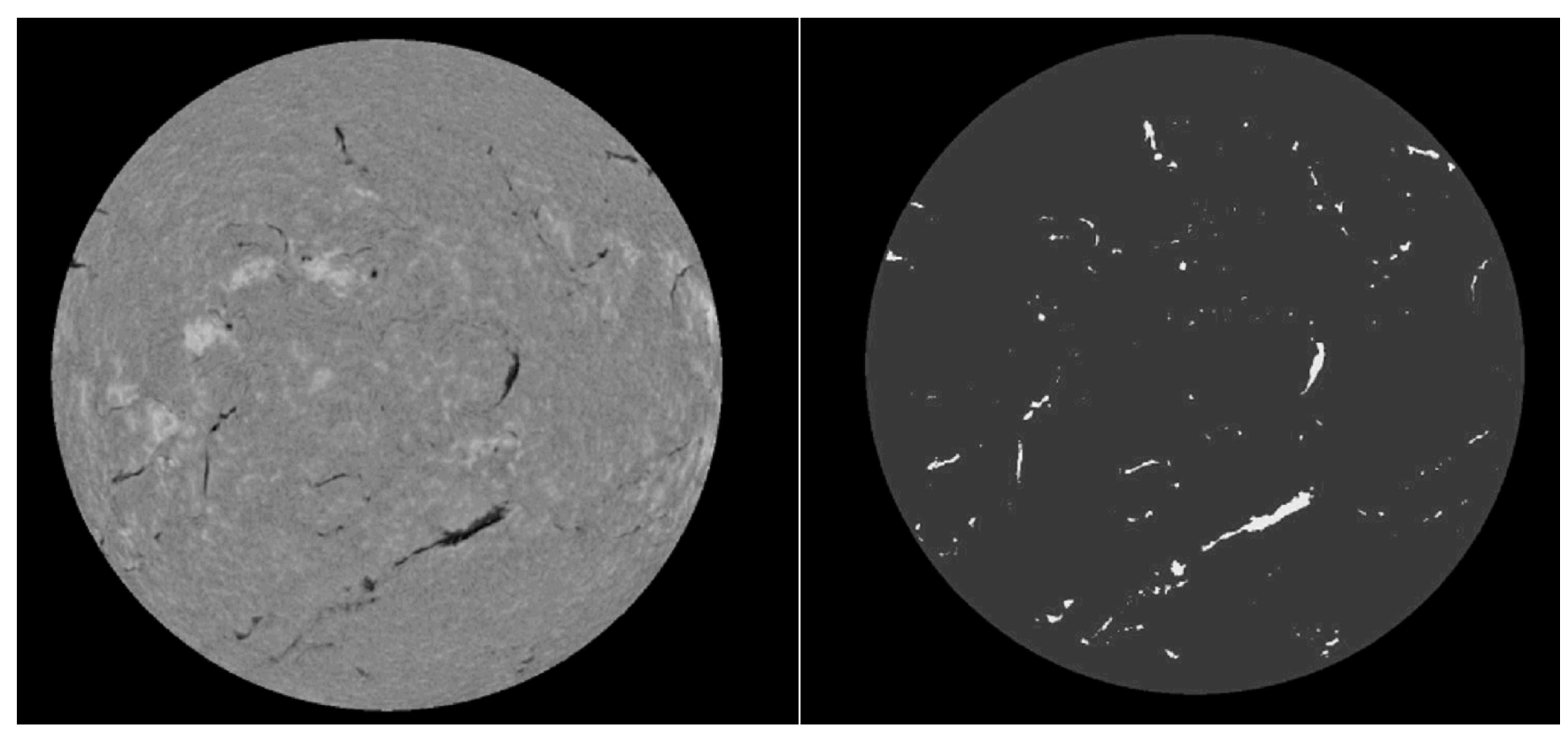

Fig. 1. Example of automatic filament detection. Left image is the original Meudon $\mathrm{H} \alpha$ spectroheliogram. Right image shows detected filaments.

looking at those maps, interesting periods can be easily detected during one rotation, such as maximum of activity for faculae, periods of stability or instability for filaments. Some studies based on synoptic maps are described in Mouradian (1998). The examples are about detection of 'pivot-points' and emerging sunspots in pre-existing plages.

\subsection{Overall description}

Shown in Fig. 2 is the behavior of plages, sunspots and filaments plotted on Meudon Synoptic Maps of Solar Activity. It is critical to notice that the actual plot does not correspond to the actual position of solar features, however it is a synthesis of their behavior. Synoptic maps represent one solar rotation plotted on a graph with latitude as ordinate, and Carrington longitude, or equivalently time, as abscissa. On those maps, available through the BASS2000 database ${ }^{4}$, plages are indicated in red, sunspots in blue and filaments in green. Plages that are too scattered, and one-day filaments, are not represented. Furthermore, only sunspots larger than $2000 \mathrm{~km}$, which can be detected on images, are plotted on these maps. Thus, features are represented using the following protocol:

- In an active region, plages are separated using the magnetic polarity of sunspots and faculae. Thus, these active centers are plotted at the maximum of their evolution, corresponding to the maximum of their spotted area.

\footnotetext{
${ }^{4}$ http://bass2000.obspm.fr/home.php choose "For the synoptic maps" in the left panel
}

- Plages without any spots are plotted at the maximum of their surface.

- Filaments are plotted at their maximum extension. The drawing corresponds to their mean position during the rotation considered (see Sect. 4.2 for details).

Hatches complete the description of features on synoptic maps. When tight, they indicate:

- very bright faculae,

- stable filaments (i.e. number of days the filament stayed at the same place).

Finally, blue dots represent sunspots with a size proportional to their diameter.

\subsection{Dedicated filament maps}

On those Carrington maps (see the example in Fig. 3), the simplified skeleton of filaments is plotted daily. At the end of the rotation, envelopes of groups of close filament strokes are drawn. As a result, it can be deduced that all strokes from one group are the daily representation of the same filament. From this technique, the persistence of a filament can also be deduced: actually, the more strokes at the same position, the more long-lived the filament is. This information leads to a synthesized filament appearing on the Synoptic Maps of Solar Activity. 


\section{ROTATION 2008}

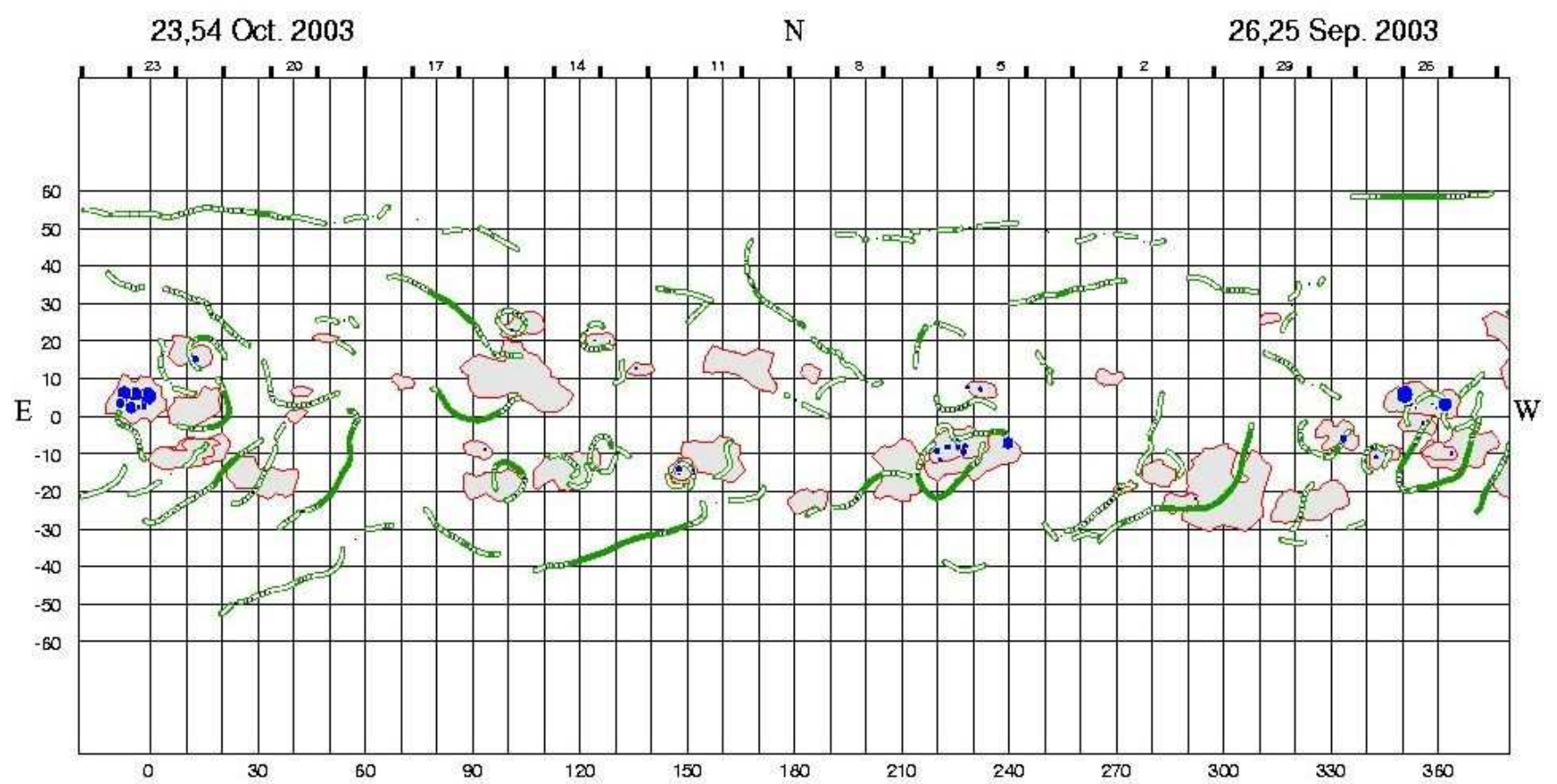

Fig. 2. Example of a Meudon Synoptic Map of Solar Activity.

\section{Automatic generation of synoptic maps of filaments and their tracking}

Since the shape of filaments may change, their position may vary, and sky transparency can interfere, keeping track of a filament automatically during a rotation is not easy to achieve. Therefore, to solve this difficulty an original approach has been adopted. The overall process is shown in Fig. 4.

The principle of the tracking is based on the process that was manually made to produce the synoptic maps, namely the grouping of intersecting envelopes of filaments. However, to automate the generation of synoptic filament maps, more sophisticated processing must be added. After having placed skeletons and envelopes of filaments on a Carrington map, a region-growing type algorithm is applied (Cao and Collin, 2001) and intersection of surfaces can be computed. The next step is to compute a regression curve for and from all groups. Then, discretizers are setup to evaluate the persistence of the filament: segments of straight lines normal to this regression are placed at regular curvilinear intervals on the curve. The total number of filaments crossing each discretizer is computed indicating its degree of persistence. From this information, the shape of the synthesized filament is deduced (full green, half-full and empty areas).
Two types of regression curves are needed depending on the aspect of the filament. A polynomial regression is computed for near-linear shapes whereas elliptic regression is used for near-elliptic or circle shapes.

The use of intermediate synoptic maps for feature tracking enables the rejection of feature distortion (real or due to instruments) that often prevents this kind of study. It allows to show connections between apparently separated features (even when features appear to split or to merge). Moreover, this technique can be applied to several structures in visible wavelengths, e.g. sunspots and active regions. Several UV features can also benefit from such a process.

The few processed examples give promising results but we still have to check the method against filaments having an unusual behavior. Hence, we plan to process the time periods corresponding to the existing Meudon synoptic maps and compare our results with the original manual synthesis.

\section{Examples of scientific investigations using Meudon synoptic maps}

While synoptic coronal hole maps were initiated at the National Solar Observatory for studies related to the solar wind, Meudon Synoptic Maps were created earlier, in 1928, to monitor and investigate solar activity. Such maps are also 


\section{ROTATION 2008}

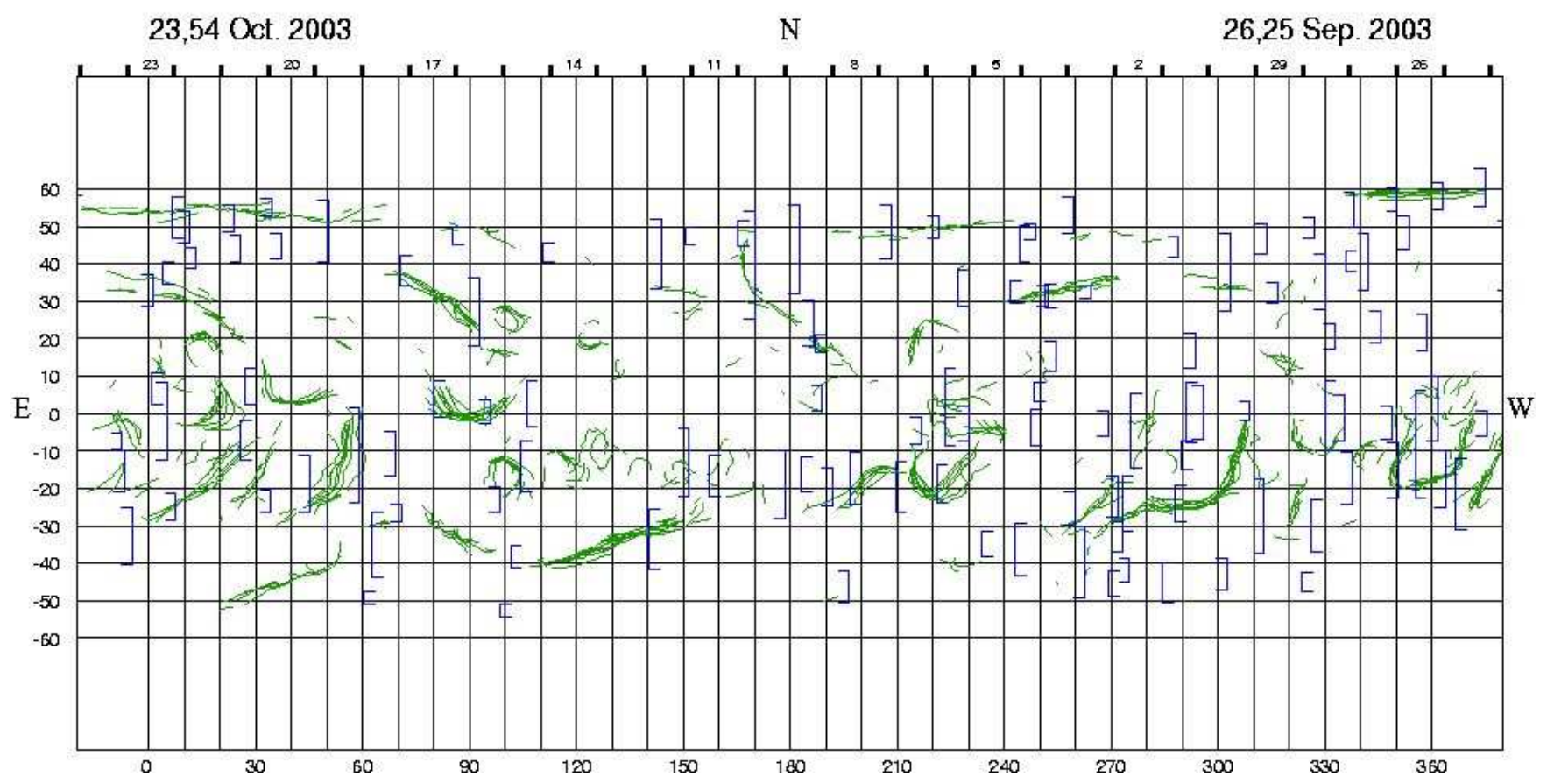

$S$

Fig. 3. Example of a Meudon Synoptic Map dedicated to filaments. Each stroke corresponds to the daily position of each filament. The drift or bending of any filament can be directly seen on this map. The blue square brackets correspond to filaments observed off the limb, which are not considered in this article. See text for details.

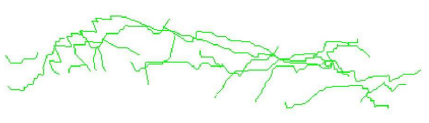

Initial daily tracks

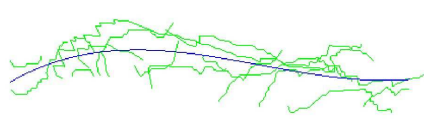

Regression curve

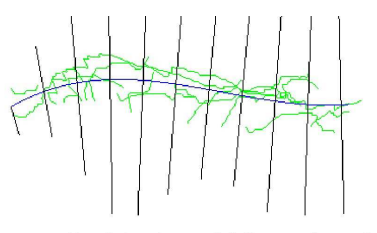

Positioning of 'discretisers'

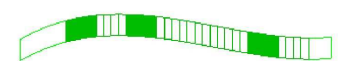

Final synthesized filament

Fig. 4. Sequence of steps describing the method used to compute the synthesis of filament behavior.

valuable tools for the investigations of the behavior of filaments as a function of time. The significance of filaments has been already demonstrated as they are known to be one of the sources of CMEs or flares. A better understanding of filament evolution could help the prediction of CMEs, for example. Moreover, other investigations rely on the behavior of filaments as a function of time, such as the sun magnetic field, as well as streamers and their evolution.

We present in this section a few examples of recent investigations that extensively used Meudon Synoptic Maps as an observing tool to study filament evolution through time.

As already mentioned in Sect. 2, Mouradian and SoruEscaut (1989) used a manual tracking method to explain how sudden disappearances of solar filaments are only temporary as they can be still seen in UV, and therefore are thermal phenomena.

In the statistical analysis of the magnetic field in the vicinity of filaments, Ipson et al. (2005) conclude their work by expressing the need for a feature tracking method for a better understanding of solar activity.

Rust and LaBonte (2005) show that MHD helical kink instability is associated with solar eruptions. They mention several characteristics of an erupting filament (e.g. longduration, pattern of barbs) that may be used in further studies - assuming a tracking of filaments is available - to determine if those characteristics can be an indicator for a flare to come, thus providing a new tool for flare prediction. 
Another example of filaments and flares study is the one presented by Isobe and Tripathi (2006) who show that filaments are often related to solar flares. Several specific behaviors have been detected shortly before their eruption. Filament tracking, as proposed in our paper, will give the opportunity to determine if filaments follow a typical behavior before they erupt, or if this is related to other signatures of activity on the Sun. This will represent a significant advance in the understanding of solar flares.

Wang et al. (2006) show correlation between interplanetary magnetic clouds and associated filaments. This study is based on three events. The existence of a database including tracking information will give the opportunity to extend statistics to more significant numbers.

Finally, Morgan and Habbal (2007) show that large-scale coronal structures (helmet streamers) can be the result of the convergence of two or more sheet-like structures originating from a distribution of filaments on the solar disk. A longterm study of filaments distribution on the solar disk could help to confirm this result.

\section{Conclusions}

We show here that a method inherited from image processing techniques can be used to solve the difficult problem of time tracking of filaments. The advantage of this method consists not only of its ease, but also of the possibility to overcome the problems related to observations, such as segmentation of filaments for a time period, change in atmospheric transparency, or one or two days of filament disappearance. There is another value to this method: it can be applied to any structure stable enough to remain at the approximate same location during a Carrington rotation. Furthermore, it is then possible to determine in some cases where structures come from. For instance, if a sunspot splits in several parts, it will appear on the Carrington map as one region that may be identified quite clearly.

Nevertheless, one must be cautious, as an extensive check of the validity of this method was not made. In particular, one must be sure that two filaments in close proximity during a rotation do not merge into one, or that a complicated filament shape is not interpreted as two separate ones. Once this method is extensively tested as well as extended to sunspots and active regions, we plan to resume the publication of Meudon synoptic maps. We expect also to provide not only those maps for each Carrington rotation, but also shifting maps that may be drawn at will for a requested period of time. If the first results presented here are confirmed, this technique will address and solve an important issue in the entries of filament catalogs with information concerning their time evolution.
Acknowledgements. Topical Editor R. Forsyth thanks J.-C. Noëns and another anonymous referee for their help in evaluating this paper.

\section{References}

Aboudarham, J. and Zharkov, S.: Features Parameters Description and Organization: Features Parameters Description and Organization,, Tech. rep., http://www.egso.org/documents/Features_ ParametersV2.3.pdf, 2004.

Benkhalil, A., Zharkova, V. V., Ipson, S., and Zharkov, S.: Active Region Detection and Verification With the Solar Feature Catalogue, Solar Phys., 235, 87, doi:10.1007/s11207-006-0023-7, 2006.

Bentley, R., Csillaghy, A., and Scholl, I.: The European Grid of Solar Observations, in: Proceedings of the SPIE, vol. 5493, pp. 170-177, 2004.

Cao, F. and Collin, B.: Region tracking using level set registration, in: Proceedings of SPIE, vol. 4365, pp. 102-111, 2001.

Fuller, N., Aboudarham, J., and Bentley, R.: Filament Recognition and Image Cleaning on Meudon Halpha Spectroheliograms, Solar Phys., 227, 61, doi:10.1007/s11207-005-8364-1, 2005.

Ipson, S. S., Zharkova, V. V., Zharkov, S., Benkhalil, A. K., Aboudarham, J., and Fuller, N.: Automated Technique For Comparison Of Magnetic Field Inversion Lines With Filament Skeletons From The Solar Feature Catalogue, Solar Phys., 228, 399421, doi:10.1007/s11207-005-6878-2, 2005.

Isobe, H. and Tripathi, D.: Large amplitude oscillation of a polar crown filament in the pre-eruption phase, Astron. Astrophys., 449, L17-L20, doi:10.1051/0004-6361:20064942, 2006.

Morgan, H. and Habbal, S. R.: An empirical 3D model of the large-scale coronal structure based on the distribution of $\mathrm{H} \alpha$ filaments on the solar disk, Astron. Astrophys., 464, 357-365, doi: 10.1051/0004-6361:20066482, 2007.

Mouradian, Z.: Synoptic Data Findings, in: ASP Conf. Ser. 140: Synoptic Solar Physics, edited by: Balasubramaniam, K. S., Harvey, J., and Rabin, D., vol. 140, p. 197, 1998.

Mouradian, Z. and Soru-Escaut, I.: Role of rigid rotation in the sudden disappearance of solar filaments, Astron. Astrophys., 210, 410-416, 1989.

Rust, D. M. and LaBonte, B. J.: Observational Evidence of the Kink Instability in Solar Filament Eruptions and Sigmoids, Astrophys. J. Lett., 622, L69-L72, doi:10.1086/429379, 2005.

Wang, Y., Zhou, G., Ye, P., Wang, S., and Wang, J.: A Study of the Orientation of Interplanetary Magnetic Clouds and Solar Filaments, Astrophys. J., 651, 1245-1255, doi:10.1086/507668, 2006.

Zharkov, S., Schetinin, V., and Zharkova, V.: The Automatic Classification with ANN of sunspot groups using the Solar Feature Catalogue, in: 35th COSPAR Scientific Assembly, p. 2331, 2004.

Zharkova, V. V., Ipson, S. S., Zarkov, S. I., Benkhalil, A., Aboudarham, J., and Bentley, B.: A full-disk image standardisation of the synoptic solar observations at the Meudon Observatory, Solar Phys., 214, 89, doi:10.1023/A:1024081931946, 2003. 